\title{
L'intelligence du corps
}

Gilles Bui-Xuân, Jacques Gleyse et Eric De Léséleuc

\section{CpenEdition}

Journals

Édition électronique

URL : http://journals.openedition.org/trema/2444

DOI : $10.4000 /$ trema.2444

ISSN : 2107-0997

Éditeur

Faculté d'Éducation de l'université de Montpellier

Édition imprimée

Date de publication : 24 octobre 1997

Pagination : 1-5

ISSN : 1167-315X

Référence électronique

Gilles Bui-Xuân, Jacques Gleyse et Eric De Léséleuc, «L'intelligence du corps », Tréma [En ligne], Hors série $N^{\circ} 1$ | 1997, mis en ligne le 29 septembre 2015, consulté le 22 septembre 2020. URL : http:// journals.openedition.org/trema/2444 ; DOI : https://doi.org/10.4000/trema.2444

Ce document a été généré automatiquement le 22 septembre 2020.

Trema 


\title{
L'intelligence du corps
}

\author{
Gilles Bui-Xuân, Jacques Gleyse et Eric De Léséleuc
}

1 Ce numéro de Tréma est doublement spécial. Il se distingue en effet des numéros habituels parce qu'il publie, comme ce fut le cas pour la biologie, les actes d'un colloque et que, de ce fait, il n'est pas semblable au volume habituel de la revue. Il s'en distingue également parce qu'il est constitué d'une partie «papier» conformément aux numéros précédents, mais aussi, et c'est surtout là que réside l'innovation : d'un CD-Rom.

2 la partie papier propose au lecteur les conférences plénières du colloque, la deuxième (c'est ainsi que le CD-Rom a été pensé) permet de publier (ce qui n'aurait pas été possible en volume) non seulement la totalité des communications proposées, mais aussi quantité d'images intégrées, des parties d'allocutions d'ouverture, des extraits filmés des ateliers de pratique- ce qui était une originalité du colloque-, des «fricassées » (pour l'exprimer comme Montaigne) de forums et des images d'ambiance. Bref si la première partie est plutôt traditionnelle, la deuxième utilise à plein un nouveau médium de publication qui semble particulièrement bien adapté au monde des pratiques corporelles. Si la forme de publication des actes du colloque est donc originale, le fond ne l'est pas moins.

3 « Comment peut-on enseigner une culture corporelle?» telle est l'interrogation qui servit de thème au colloque organisé conjointement par le Laboratoire Corps et Culture de l'Université de Montpellier I et le département E.P.S. de l'IUFM de Montpellier, et soutenu par l'AFRAPS, la Région Languedoc-Roussillon, et le Département de l'Hérault. Cette interrogation est à la fois provocatrice et paradoxale.

4 Provocatrice, car elle suppose a priori qu'une culture corporelle peut s'enseigner, ce qui évidemment conduit immédiatement tout sociologue ou anthropologue à se récrier: "une culture quelle qu'elle soit ne s'enseigne pas, elle se transmet». Autrement dit, il est particulièrement difficile de savoir comment s'opère le passage de l'adulte à l'enfant, d'une génération à une autre ou d'un "sujet-supposé-cultivé » à un « sujet-supposé-inculte ». 
5 Provocatrice aussi, car elle renvoie d'évidence à une rediscussion approfondie du présupposé stéréotypal, véhiculé dans le champ de l'E.P.S., selon lequel il y aurait des activités «culturelles de référence » (sous-entendu, les sports de compétition institutionnalisés et règlementés, ou les danses répertoriées) et d'autres qui ne le seraient pas (par exemple les jeux traditionnels, les activités informelles, les activités inventées par les enseignants, les activités non motrices...). Globalisant et unifiant la notion de culture, la question initiale présuppose de définir une culturalité de référence face à d'autres éléments discriminés comme non culturels. Elle pose également, d'emblée, face à ce problème, la question de la mobilité ou de l'immobilité de la notion de culture, la question de la reproduction ad vitam æternam, de pratiques closes sur elles-mêmes ou, à l'inverse, d'une dynamique permanente de la culture et, spécifiquement, de ce que l'on suppose pouvoir enseigner de la culture.

6 Mais, la question posée est également paradoxale dans la mesure où elle revient à dire que le système "culture corporelle" est tellement complexe qu'il est impossible d'en extraire les grandes lignes, l'intelligence. Dans ce cas, à quoi servirait l'école et à quoi servirait l'enseignement? (Ce n'est pas un hasard si ce colloque succède à celui de Poitiers, intitulé «â quoi sert l'EPS?») Et dans ces conditions, comment peut-on prétendre enseigner une culture corporelle? Accepter d'emblée, comme on voudrait le faire croire de Mauss ou de Bourdieu, qu'une culture se transmet seulement, suppose qu'il n'y a que société de reproduction dans laquelle aucun mouvement n'est véritablement possible et dans laquelle l'enseignement scolaire ne servirait qu'à reformuler à l'infini les mêmes catégories. Bref ce premier point de vue semble, pour les organisateurs du colloque, devoir être discuté, mais il ne peut l'être qu'en fonction de la définition que l'on souhaite donner de la notion même de culture, et qui plus est, de culture du corps.

7 La notion de culture peut être renvoyée, au-delà de son étymologie qui définit un système de circularité ou une transformation agraire de la nature, â deux visions. La première élitiste, savante, distinctive correspond plutôt à la définition donnée par A. Finkelkraut dans La défaite de la pensée. Serait véritablement culturel ce qui résiste au temps et aux modes- en caricaturant: Molière, Mozart, Le Lorrain, Van Gogh et ne serait pas culturel, le rap, le rock, le pop art, ou toute production que l'on suppose vite oubliée par l'ensemble du monde humain.

8 La deuxième correspond à la définition classique de l'ethnologie ou de l'anthropologie: est culture toute transformation, faisant sens, de la nature par l'homme. Ainsi, toute technique du corps est culturelle (dormir, manger, se nourrir, jouer, faire l'amour...) mais aussi, il ne saurait y avoir de véritable hiérarchie entre une culture des "gens de peu», comme le dit Pierre Sansot, ou une culture populaire et une culture oligarchique. Dans cette perspective, est culturel N. T.M. comme Mäbler, le hip-hop comme le Taï-Ji-Quan, le cassoulet comme un poème de Nazim Hikmet, le street-basket comme l'athlétisme de haut-niveau, la belote comme le Go. Dans cette deuxième acception, la réponse à la question posée par le colloque devient d'une complexité telle que toute velléité de réponse monovalente est réduite à néant. Le réel culturel devient en notre monde incommensurable et donc presque irréductible à un enseignement, à moins d'opérer des choix radicaux.

9 Pourtant, lorsque l'on observe le contenu des communications, qu'il n'est pas possible de résumer ici, puisqu'elles sont au nombre de 52 , plénières et ateliers 
compris, on constate que deux types au moins de réponses apparaissent. Le premier, plutôt pragmatique, consiste â entériner l'idée que l'on peut enseigner une culture corporelle, ou plutôt ne s'interroge pas vraiment sur le sujet et admet implicitement que cela est possible, en faisant part de propositions concrètes. Le deuxième type de réponse, plutôt épistémologique ou sociologique, récuse a priori l'idée d'un possible enseignement d'une culture corporelle ou du moins le rediscute vivement. Pourtant, ce deuxième type de réponses conduit presque toujours à l'idée que certaines zones de cet univers infini sont susceptibles d'être enseignées. Autrement dit, si le point de vue défendu est l'aporie de la question posée par les organisateurs, on perçoit que finalement, en ordonnant davantage, en régularisant certains lieux, en les soumettant à un modèle ou à une classification, des îlots de culture corporelle sont peut-être susceptibles d'être enseignés, comme s'ils émergeaient d'une intelligence du corps.

Il reste alors, ce qui n'a été qu'ébauché, à définir des cadres et des modèles d'intelligibilité qui permettraient de savoir, dans les océans de faits culturels (corporels), ce qui relèverait plutôt d'un enseignement et ce qui serait tributaire d'une imprégnation, d'une diffusion ou de tout autre mode de transmission. Cela est très loin d'être négligeable pour la constitution de programmes scolaires ou pour l'intégration de populations étrangères. L'idée de «volume culturel » contraindrait immédiatement l'enseignant à se questionner sur l'optimisation de celui-ci, et par là sur les véritables effets de sa propre intervention.

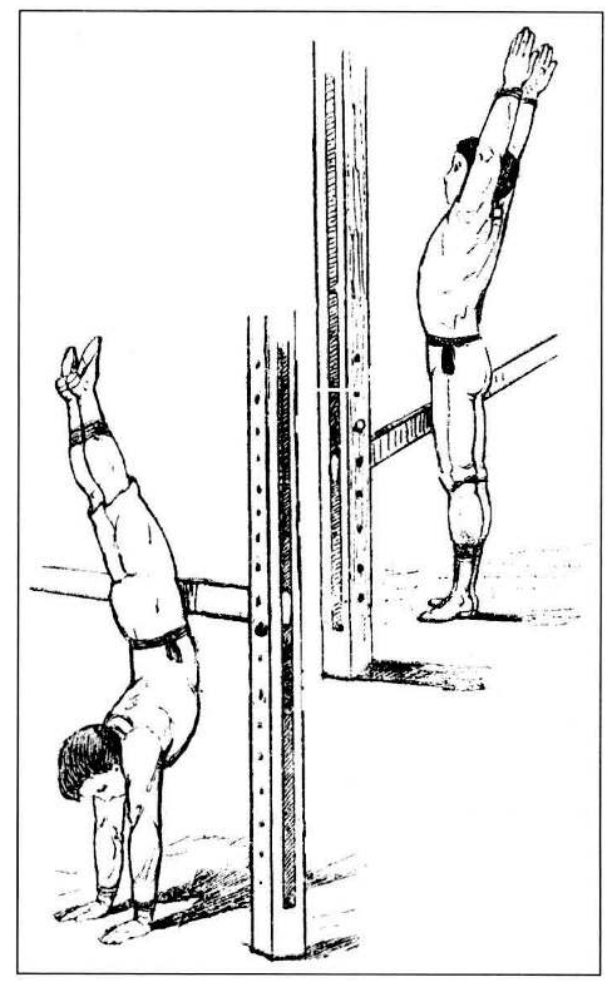

ILLUSTRATION PROVENANT d'Un MANUEL DE gYMNASTIQUe AU TOURNANT dU SIÈCLE. Page 103

Figures 18 \& 18 bis. «Passage de la position abdominale debout à la position abdominale renversée, mains sur terre. Pour exécuter ce mouvement [...] faire une grande flexion du corps en avant, en posant les mains à terre à écartement des épaules, les jambes étendues [...]. » SOLEIROL de SERVES (médecin-gymnaste, professeur de gymnastique suédoise au lycée de jeunes filles de Versailles) \& Le ROUX (professeur de gymnastique au lycée de Versailles), Manuel de Gymnastique Rationnelle et Pratique (méthode suédoise), Paris, Masson et Cie éditeurs, 1905. 
11 Ensuite, il est nécessaire d'effectuer un constat sur les types et les catégories d'ateliers et de communications présentés. Seuls, alors que le reproche est souvent fait aux colloques de ne pas allier le "dire et le faire ", six ateliers de pratique ont été proposés. Si cela représente une avancée par rapport â la plupart des colloques en E.P.S., cette raréfaction du discours corporel pratique est pourtant, si l'on veut bien y regarder de plus près, un excellent analyseur de la situation du corps dans l'école. Quoi qu'il en soit, les lecteurs pourront, pour la première fois, trouver des extraits vidéo de ces ateliers dans le CD-Rom joint à ce numéro hors-série de Tréma. Ceci n'aurait pas été possible sans ce nouveau support qui laisse augurer de suites prometteuses.

12 Il est de même très surprenant, eu égard au thème du colloque et lorsque l'on observe l'ensemble des communications, de constater que deux grandes catégories (pourtant présentes dans la plaquette initiale) sont carrément absentes, respectivement : les apports des sciences de la vie et de la matière, et la psychologie. Comme si l'on voulait nous persuader que la culture n'a pas d'inscription dans la chair(e)... Comme si l'on voulait nous persuader que la culture ne s'inscrit pas dans l'esprit... Ce point pourtant est largement contesté par le cognitiviste Jérôme Bruner puisque selon lui non seulement «[...] la culture donne forme à l'esprit", mais encore «l'éducation » est une " entrée dans la culture". Et la boucle se referme si l'on pense avec Varella (et coll.) à « l'inscription corporelle de l'esprit ».

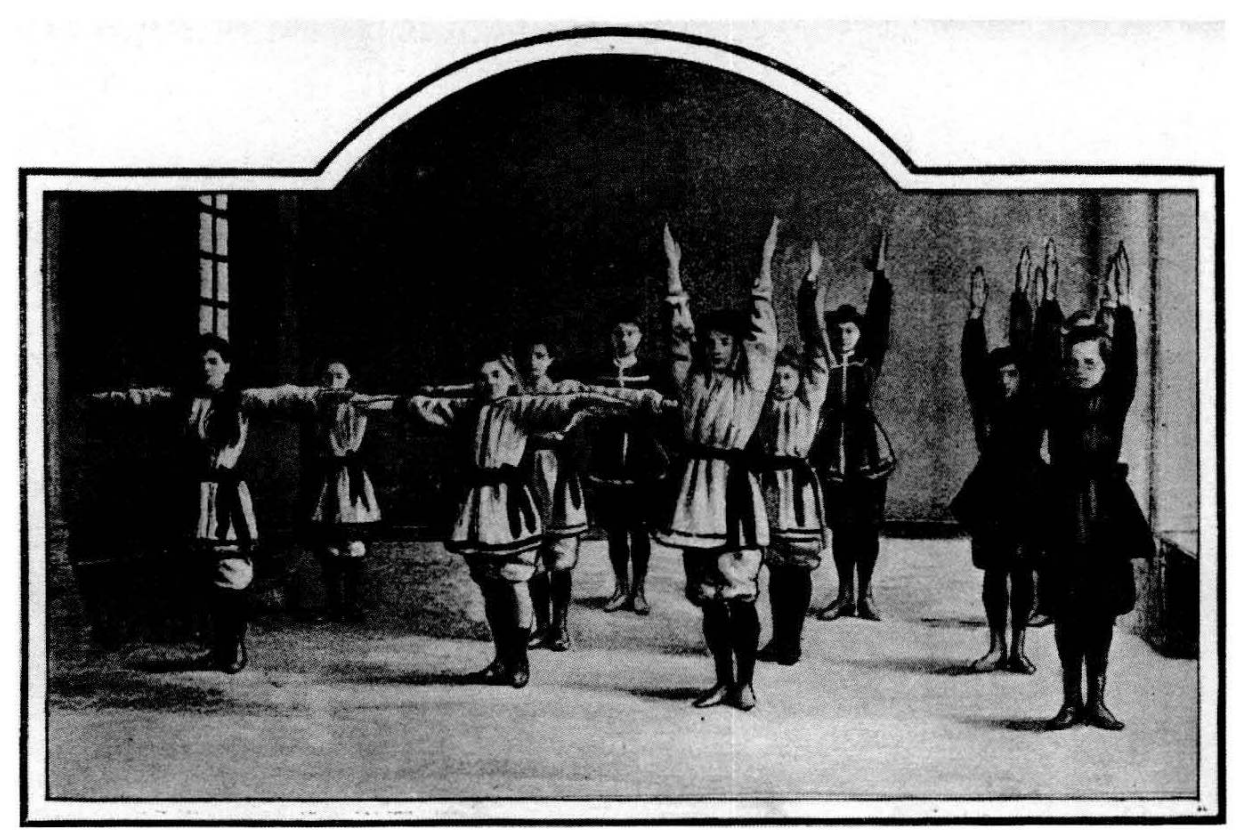

ILLUSTRATION PROVENANT d'Un MANUEL DE gYMNASTIQUE DU TOURNANT du SIÈCLE. Page 21

Chapitre III. Mouvements des bras. "Vue prise dans un lycée de l'Académie de Paris ». SOLEIROL de SERVES (médecin-gymnaste, professeur de gymnastique suédoise au lycée de jeunes filles de Versailles) \& Le ROUX (professeur de gymnastique au lycée de Versailles), Manuel de Gymnastique Rationnelle et Pratique (méthode suédoise), Paris, Masson et Cie éditeurs, 1905.

13 A moins que d'autres enjeux expliquent cette absence, que l'on doit regretter pour l'intérêt général.

14 Dans le même sens encore, comment peut-on expliquer la quasi absence des « didacticiens des A.P.S. »? Déficit d'information? Volonté délibérée? 
15 Points positifs, â l'inverse, l'on doit se féliciter de la présence de presque trois cents congressistes que l'on ne peut que remercier ici de leur présence et surtout de leur dynamisme et de leur implication active dans l'événement (par exemple au cours des forums et des ateliers de pratique). Sans eux, bien évidemment, rien n'aurait pu se faire. Dans le même sens, la présence de plusieurs Canadiens et Belges, de Suisses, d'Italiens, d'Allemands, d'un Danois et même d'une Japonaise témoignent de l'intérêt international suscité par cette manifestation.

16 La présence enfin, à la même tribune lors de l'ouverture du colloque, du Doyen de la Faculté des Sciences du Sport et de l'Education Physique, du Directeur de l'Institut Universitaire de Formation des Maîtres et de l'Inspecteur Pédagogique Régional d'E.P.S. montre les relations de bonne intelligence qui peuvent se nouer autour d'un tel questionnement et de telles manifestations.

17 La participation de nombreux étudiants a également permis la réussite du Colloque et s'il n'est pas possible de citer ici tous ceux qui ont « mis la main à la pâte » (leurs noms figurent à la page des remerciements personnalisés du CD-Rom), ils trouveront là l'expression de toute la gratitude des organisateurs pour leur compétence, leur capacité d'initiative et leur dévouement.

18 Enfin, il n'est pas possible de passer sous silence le laboratoire Corps et culture et les membres du comité scientifique qui ont accepté d'être les garants scientifiques de cette manifestation.

19 Le lecteur trouvera donc, successivement, dans ce numéro un peu particulier de Tréma, la liste exhaustive des ateliers et des communications du colloque, publiés dans le CD-Rom, les articles correspondant aux six conférences plénières avec leurs résumés en français et en anglais, un CD-Rom (qu'il sera aisé d'utiliser dans les institutions des participants si ceux-ci ne possèdent pas personnellement de lecteur) contenant toutes les communications classées par thèmes et par auteurs, et enfin, de rapides consignes de mise en route. Rapides, parce que déjà imprégnées d'une nouvelle culture. Ce sont les corps qui utilisent les derniers média de communication, plus intuitifs et plus sensitifs. et nous faisons le pari du bon usage de l'intelligence du corps. 